\title{
Impact of SARS-CoV-2 On St-Elevation Myocardial Infarction (STEMI)
}

\author{
Fatemeh-Sadat Mahdavi, Armin Khavandegar and Parham Mardi* \\ Student Research Committee, Alborz University of Medical Sciences, Karaj, Iran \\ *Corresponding author: Parham Mardi, Student Research Committee, Alborz University of Medical Sciences, Karaj, Iran. \\ To Cite This Article: Parham M, Fatemeh-Sadat M, Armin K. Impact of Sars-Cov-2 On St-Elevation Myocardial Infarction (Stemi). 2020 - 9(1). \\ AJBSR.MS.ID.001355. DOI: 10.34297/AJBSR.2020.09.001355.
}

Received: 眥 May 01, 2020; Published: 阱 June 02, 2020

\section{Introduction}

Severe Acute Respiratory Syndrome-Corona Virus-2 (SARS-CoV 2) pandemic originated from Wuhan, China, in December 2019 and rapidly spread throughout the globe [1]. Currently, SARS-CoV 2 has resulted in an enormous number of deaths worldwide [2,3]. Based on the WHO report on 28 April 2020, almost 3 million confirmed cases of COVID-19 have been identified, and nearly 7\% of them have died due to disease and its complications [4]. Regarding the catastrophic impact of COVID-19 on human well-being, investigation of its manifestations, complications, and appropriate treatment has become the most concerning issue in the World Health Organization recently.

Elders, especially those with underlying diseases, including chronic bronchitis, congestive heart diseases, and diabetes, are more susceptible to COVID-19 complications [5]. Various types of complications with different prevalence have been reported in literature consisting of Neurologic [6], Hematologic (including coagulative disorders) [7], Hepatic [8], renal [9], and finally Cardiovascular complications [10].

Patients suffering from COVID-19 are mostly presented with respiratory involvement, fever, and myalgia; Generally, respiratory involvement and general condition exacerbate and eventually leads to pneumonia and, in severe cases, results in Acute Respiratory Distress Syndrome (ARDS) or shock. The most common manifestations revealed in COVID-19 patients are fever, cough, and malaise [11]. Compared to respiratory involvement, cardiovascular findings are less encountered at admission or hospitalization; Nevertheless, studies reminded a considerable impact of cardiovascular involvement in COVID-19 patients on disease prognosis.

Cardiovascular involvement, defined as a considerable rise in at least one of biomarkers, is associated with COVID-19 poor prognosis [10]. In this issue, Ruan et al. claimed that the total number of patients who died of myocardial damage/heart failure with or without respiratory failure comprised $40 \%$ of all deaths. (3) Other studies indicated that the mortality rate in COVID-19 patients complicated with acute heart failure is conspicuously higher in comparison with other complications and risk factors, including elderly, diabetes, chronic pulmonary diseases, and history of cardiovascular diseases. Despite the high prevalence of COVID-19, little information exists about the cardiac involvement of this disease.

Various cardiac manifestation has been reported in COVID-19 patients consisting of cardiogenic shock, Acute myocardial damage, ST-elevation Myocardial Injury, Viral Myocarditis, Arrhythmiainduced cardiomyopathy, and venous thrombosis [12-14]. A caseseries in Washington D.C. revealed that 33\% of COVID-19 patients in the Intensive Care Unit have experience cardiomyopathy [15]. Besides, there were reports of cardiac Tamponade and Takotsubo cardiomyopathy secondary to COVID-19 [16]. The physiopathology behind the cardiac damage in COVID-19 is not still clear; however, scientists believe that Plaque rupture, Cytokine storm, inflammatory damage, hypoxic damage, coronary spasm, micro thrombus, and direct endothelial damage probably play important roles [17].

Cardiac symptoms in COVID-19 patients could be found in ECG, while no other findings are presented. In this regard, a 66-yearold Chinese woman with "temporary S1Q3T3 morphology" and secondary "reversible nearly complete A.V. block" without any remarkable past medical history has been reported. Furthermore, many cases of COVID-19 patients with ST-elevation have been registered. ST-elevation Myocardial Infarction is one of the most important causes of death due to cardiovascular involvement. Generally, patients with NSTEMI have a better prognosis than STEMI [18]. 
The first COVID-19 patient with simultaneous STEMI was a 31-year-old female with remarkable past medical history comprising of COPD, Coronary artery disease, Pulmonary Hypertension, Diabetes, and CVA. She denied any history of fever, sore throat, and G.I. discomfort. In basic ECG on admission, STelevation in Leads 2, 3, and AVF with reciprocal changes in V1 and V2 were demonstrated, which is determinant of Acute Inferior Wall STEMI. She underwent Coronary revascularization and successfully cured [19].

Another surprising report about the cardiac manifestation of COVID-19 was a 70-year-old patient with a history of Hypertension and D.M. type-2 hospitalized with COVID-19 impression; Despite appropriate treatment, bilateral pulmonary infiltration progressed, and Hypoxia deteriorated. During the hospitalization, she experienced ST-elevation accompanied by multifocal ventricular tachycardia and, unfortunately, died 24 hours later [5]. Another study reported 64 years old woman with no fever and pulmonary symptoms with a history of Hypertension and Hyperlipidemia, who was presented with Acute chest pain on admission. Performing ECG, ST-elevation, and Sinus tachycardia were discovered. Following angiography exposed non-obstructive CAD [14].

In a case-series study through the COVID-19 outbreak in the United States, 18 cases with ST-elevation were reported. Ten of them were hospitalized with ST-elevation on admission, while eight others developed ST-elevation during the hospitalization. The average age was 63(54-73) (median and interquartile range). $18 \%$ of patients had a history of CAD who had a poor prognosis and different manifestations compared to others. Coronary angiography proceeded for $50 \%$ of cases. $66 \%$ of angiography results indicated coronary obstruction. Thirteen patients died due to cardiac complications [20].

Various Guidelines have evolved for approaching to COVID-19 patients with Acute Myocardial Damage [8,15, 21,22]. As a result of the presence of numerous differential diagnoses for ST-elevation, including viral myocarditis, the determination of appropriate treatment has remained a challenge; Nonetheless, primary PCI is the standard treatment in STEMI patients. Fibrinolysis-based strategies are the substitutive treatment in the absence of PCI equipment [23].

Many recommendations have been suggested for the protection of health providers during Cardiovascular procedure in the COVID-19 pandemic, including the provision of essential services and minimizing any chance of COVID-19 spreading [24,25].

Due to poor prognosis and disastrous consequences of COVID-19 patients with simultaneous cardiac involvement or secondary cardiac complication and reports of STEMI in COVID-19 patients with no remarkable medical history, we highly recommend our colleagues throughout the globe to perform ECG in COVID-19 patients as soon as possible, no matter what the medical history or general condition is.

Further studies are needed to investigate the effect of COVID-19 on Cardiovascular function. We suggest physicians be thoroughly informed of atypical findings of COVID-19, early diagnosis, and treatment approaches.

\section{References}

1. Yang X, Yu Y, Xu J, Shu H, Liu H, et al (2020) Clinical course and outcomes of critically ill patients with SARS-CoV-2 pneumonia in Wuhan, China: a single-centered, retrospective, observational study. The Lancet Respiratory Medicine 8(5): 475-481.

2. Mahase E (2020) China coronavirus: WHO declares international emergency as death toll exceeds 200. BMJ 368: m408.

3. Ruan Q, Yang K, Wang W, Jiang L, Song J (2020) Clinical predictors of mortality due to COVID-19 based on an analysis of data of 150 patients from Wuhan, China. Intensive care medicine 46(5): 846-848.

4. WHO (2020) Situation report - 98.

5. Pan L, Mu M, Yang P, Sun Y, Wang R, et al. (2020) Clinical characteristics of COVID-19 patients with digestive symptoms in Hubei, China: a descriptive, cross-sectional, multicenter study. The American journal of gastroenterology. 115(5): 766-773.

6. Filatov A, Sharma P, Hindi F, Espinosa PS (2020) Neurological complications of coronavirus disease (COVID-19): encephalopathy. Cureus 12(3): e7352.

7. Terpos E, Ntanasis Stathopoulos I, Elalamy I, Kastritis E, Sergentanis TN, et al. (2020) Hematological findings and complications of COVID-19. American Journal of Hematology.

8. Zhang C, Shi L, Wang FS (2020) Liver injury in COVID-19: management and challenges. The Lancet Gastroenterology \& Hepatology 5(5): 428430 .

9. Cheng Y, Luo R, Wang K, Zhang M, Wang Z, et al. (2020) Kidney disease is associated with in-hospital death of patients with COVID-19. Kidney international 97(5): 829-838.

10. Shaobo S, Qin M, Shen B, Yuli Cai, Tao Liu, et al. (2020) Association of Cardiac Injury With Mortality in Hospitalized Patients With COVID-19 in Wuhan, China. JAMA cardiology e200950.

11. Hasani H, Mardi S, Shakerian S, Taherzadeh-Ghahfarokhi N, Mardi P (2020) The Novel Coronavirus Disease (COVID-19): A PRISMA Systematic Review and Meta-analysis of Clinical and Paraclinical characteristics. medRxiv.

12. Fried JA, Ramasubbu K, Bhatt R, Topkara VK, Clerkin KJ, et al. (2020) The variety of cardiovascular presentations of COVID-19. Circulation.

13. Long B, Brady WJ, Koyfman A, Gottlieb M (2020) Cardiovascular complications in COVID-19. The American Journal of Emergency Medicine.

14. Juusela A, Nazir M, Gimovsky M (2020) Two Cases of COVID-19 Related Cardiomyopathy in Pregnancy. American Journal of Obstetrics \& Gynecology 2(2): 100113.

15. Guo T, Fan Y, Chen M, Wu X, Zhang L, et al. (2020) Cardiovascular implications of fatal outcomes of patients with coronavirus disease 2019 (COVID-19). JAMA cardiology e201017.

16. Dabbagh MF, Aurora L, D’Souza P, Weinmann AJ, Bhargava P, et al. (2020) Cardiac Tamponade Secondary to COVID-19. JACC: Case Reports.

17. Tavazzi G, Pellegrini C, Maurelli M, Belliato M, Sciutti F, et al. (2020) Myocardial localization of coronavirus in COVID-19 cardiogenic shock. European Journal of Heart Failure 22(5): 911-915. 
18. Marceau A, Samson JM, Laflamme N, Rinfret S (2013) Short, and longterm mortality after STEMI versus NON-STEMI: a systematic review and meta-analysis. Journal of the American College of Cardiology 61(10): E96.

19. Siddamreddy S, Thotakura R, Dandu V, Kanuru S, Meegada S (2020) Corona Virus Disease 2019 (COVID-19) Presenting as Acute ST-Elevation Myocardial Infarction. Cureus 12(4): e7782.

20. Bangalore S, Sharma A, Slotwiner A, Yatskar L, Harari R, et al. (2020) ST-segment elevation in patients with COVID-19-A case series. New England Journal of Medicine.

21. Tam C CF, Cheung KS, Lam S, Wong A, Yung A, et al. (2020) Impact of coronavirus disease 2019 (COVID-19) outbreak on ST-segmentelevation myocardial infarction care in Hong Kong, China. Circulation: Cardiovascular Quality and Outcomes 13(4): e006631.

22. Driggin E, Madhavan MV, Bikdeli B, Chuich T, Laracy J, et al. (2020) Cardiovascular considerations for patients, health care workers, and health systems during the coronavirus disease 2019 (COVID-19) pandemic. Journal of the American College of Cardiology 75(18): 23522371.

23. Mahmud E, Dauerman HL, Welt FG, Messenger JC, Rao SV, et al. (2020) Management of Acute Myocardial Infarction During the COVID-19 Pandemic. Journal of the American College of Cardiology.

24. Wood DA, Sathananthan J, Gin K, Mansour S, Ly HQ et al. (2020) Precautions and procedures for coronary and structural cardiac interventions during the COVID-19 pandemic: guidance from Canadian Association of Interventional Cardiology. Canadian Journal of Cardiology 36(5): 780-783.

25. Welt FG, Shah PB, Aronow HD, Bortnick AE, Henry TD, et al. (2020) Catheterization laboratory considerations during the coronavirus (COVID-19) pandemic: from ACC's Interventional Council and SCAI. Journal of the American College of Cardiology 75(18): 2372-2375. 